\title{
Bacterial Disease Detection for Pepper Plant by Utilizing Deep Features Acquired from DarkNet-19 CNN Model
}

\author{
Alper ÖZCAN ${ }^{1}$, Emrah DÖNMEZ ${ }^{2 *}$ \\ ${ }^{1}$ Akdeniz University, Department of Computer Engineering, Antalya, ORCID: 0000-0002-5999-1203 \\ ${ }^{2}$ Bandırma 17 Eylül University, Department of Software Engineering, Bandırma, ORCID: 0000-0003-3345-8344
}

\begin{tabular}{|c|c|}
\hline ARTICLE INFO & ABSTRACT \\
\hline Article history: & $\begin{array}{l}\text { In recent years, computer-aided agriculture applications have been developing rapidly as a prominent } \\
\text { research area. In parallel with the developments in technology, the use of automatic systems, sensor fusion, }\end{array}$ \\
\hline Received 09 June 2021 & the internet of things, and artificial intelligence-based systems is becoming widespread in agriculture. The \\
\hline Rec. in rev. form 27 August 2021 & use of these systems allows for safer, faster, and more cost-effective operations based on human factors in \\
\hline Accepted 14 September 2021 & agricultural applications. Among these applications, there are artificial intelligence applications developed \\
\hline Available online $\mathrm{xxx}$ & $\begin{array}{l}\text { based on image processing and machine learning. Plant disease detection systems are also among these } \\
\text { artificial intelligence studies. Within the scope of this study: I. It has been ensured that the leaf images of }\end{array}$ \\
\hline Keywords: & $\begin{array}{l}\text { the pepper plant have been segmented and their features have been extracted from the pre-trained } \\
\text { convolutional neural network. II. These obtained features have been classified through the classifier }\end{array}$ \\
\hline Deep Features, CNN, DarkNet-19, & methods in order to detect bacterial disease. In the study, a total of 2475 images of pepper leaves with \\
\hline Plant Disease & 1478 healthy and 997 bacterial diseases, which are among the PlantVillage data sets, have been used. To \\
\hline & extract the features, the DarkNet-19 network model has been used as a pre-trained convolutional network. \\
\hline & $\begin{array}{l}\text { The SoftMax classifier in the last layer of the convolutional network model has been removed from the } \\
\text { network and SVM, KNN, and Decision-Tree-based classifiers are used instead of it. According to the }\end{array}$ \\
\hline & results, the level of performance achieved using the DarkNet-19 network and SVM classifier is quite \\
\hline
\end{tabular}

* Corresponding author

\section{Introduction}

The key causes for the degradation and depletion of plants/crops, both quality, and quantity-wise, are plant diseases [1]. Bacteria, fungi, and viruses are the primary cause of plant diseases, and these diseases can be detected by monitoring the leave, stem, or fruit section of the plant. It is very difficult for farmers to identify plant diseases without professional knowledge; farmers face many difficulties in the detection/identification of different plant diseases. It is important to have the expertise, information of crops, their different diseases as well as prevention measures, to make the right choices, and to choose the right efficient protection measures for the diagnosis and treatment. Previously identification and tracking of plant diseases were performed manually with the assistance of specialists in this area, this method of diagnosis of plant diseases is time-consuming, less effective. The identification of such diseases makes it possible for farmers to manage them correctly to improve agricultural productivity. A fully automated disease detection approach for plants is a vital subject of research because of its advantages, such as tracking huge field crops and identifying disease signs faster than any modifications on the plant. [2].
The most common method to detect plant diseases is image processing in the agriculture field. The main goal of image processing is to enhance the image of the plants. Multiple features are extracted from the enhanced image for further processing. Plant leaves are usually the first source of most plant diseases that can be identified. Effective image processing techniques can automatically identify plant diseases [3]. The detection of plant diseases by image processing is not a simple job because of the immense different possibilities for plant image such as noise, instance scale, color, form, etc. Normally, all methods used in this field contain two steps. Prominent features are extracted from the input images of the leaves in the first step, several researchers suggested various methods of extraction to extract features based on data mining, such as intra- and inter-block dependencies for Markov features, color-level features, texture features etc. A special classifier is used in the second step, which classifies the images as healthy or diseased images. Many machine learning methods can be used in this phase such as K-nearest neighbor (KNN) and support vector machine (SVM) [4].

Recent advancements in machine learning research presently perform efficient detection of plant disease from raw images. Currently, deep learning methods are performing better in literature especially in image 
processing. Deep learning-based models are based on neural networks. The main feature of the deep learningbased model is it extracts the features automatically. There are multiple neural networks such as CNN, RNN, FNN, ANN etc.

In recent years, $\mathrm{CNN}$ and $\mathrm{RNN}$ are extensively used in image processing especially for image classification. The hybrid model is introduced in [6] for the extraction of contextual information of plant leaves by using CNN. In [7] the authors used different pre-trained CNN models on the leaf's dataset. The findings of this study indicate that CNN is highly efficient to detect plant leaves diseases.

Pepper cultivations are one of the major crops in South Asia. One of South Asia's most remunerative farming industries is the production of pepper. The most widely consumed spice in the world is black pepper. The success of this crop is reported in the areas where the temperature is around 15-40 C [8]. Many Deep Learning models have lately been developed to evaluate various forms of plant diseases, but relatively limited work has been undertaken to detect plant diseases. It is very important to detect the disease timely to prevent further loss. This study uses deep learning methods to detect the disease in pepper plants. Further, the proposed model is using a DarkNet to detect the disease in pepper plant leaves. The DarkNet is an opensource neural network framework that provides extensive usability in deep learning models.

\section{Literature Review}

In this section, we will discuss some of the major studies that are present in the literature to detect plant diseases. In the literature, different machine learning and deep learning methods are used. For convenience, we can categorize the literature into two sections.

- Machine Learning Methods: Multiple machine learning methods have been used in the literature to detect plant diseases such as KNN, SVM, Decision trees, etc.

- Deep Learning Methods: The deep learning methods used in plant disease detection are CNN, RNN, ANN etc.

\section{Machine Learning Methods}

Yan-Cheng Zhang \& et al [9] proposed a fuzzy selection method on the cotton leaves. The features for this approach were selected by using fuzzy feature selection. The proposed method is divided into two steps. In the first step, the important features are automatically isolated by FC by ignoring the unnecessary features. The second step involves the fuzzy surface to obtain dependent features. This approach is used to reduce the dimensionality of the data for practical implementations.

Shiv Ram Dubey \& et al [10] proposed the K-Means clustering approach on the apple fruit for image segmentation. After that, the feature extraction on segmented images is done by Support Vector Machines. The mentioned approach is practically implemented and achieved accuracy up to $93 \%$.
Godliver Owomugisha \& et al [11] proposed a machine learning-based application that detects crop disease by capturing an image from the smartphone. The proposed system classifies the plants into 5 categories. The categories are numbered from 1-5 depending on the severity levels of the plants. The number 1 represents the healthy plant and 5 represents the diseased plant. Moreover, they have used multiple feature extraction methods to compare the performance of the model. The main goal of this study was to capture an image of the cassava plant and upload it to the server. The farmers can see the score of the plant from their smartphones.

A. Meunkaewjinda \& et al [12] proposed different artificial intelligence techniques to diagnose the grape leaf disease. The proposed work is divided into three categories; i) preprocessing using color segmentation ii) grape leaf disease segmentation iii) classification and analysis of the disease present on the leaves. Support Vector Machine is used for the classification. Three classes are created in this research; scab disease, rust disease, and no disease.

M. Ravindra Naik \& et al [13] used SVM to classify the images of the plants whether it is affected by disease or not. Moreover, the genetic algorithm is used for image segmentation. The neural network classifier is used for classification. The proposed model is tested on the banana, jackfruit, and lemon.

\section{Deep Learning Methods}

B. Liu et al proposed a deep CNN model to detect the four types of apple leaf diseases. The dataset comprised around 1100 images and was enriched by using some digital image processing techniques. The proposed model was comprised of a modified Alex-net model. The result of this study was compared with other CNN models such as Google Net and Res-Net-20 and the proposed model achieved accuracy up to $97.5 \%$ [14].

DeChant et al used a pipeline of CNN to detect the disease in the maize crop. The proposed method used multiple CNNs to classify the different regions of the leaf. After analyzing the different regions, the results are combined in the form of heat maps as an input to the final CNN for classification purposes. The dataset consisted of around 1796 images and the accuracy of the model was around $96.7 \%$ [15].

Ma et al proposed a deep convolutional neural network along with different classifiers such as Random Forest, Support Vector Machines to detect the disease in the cucumber. The dataset was collected from the Plant Village dataset, Internet websites, and other sources. Different augmentation techniques are used in this dataset. This model achieved the highest accuracy up to $94 \%$ compared to other models in the literature [16].

Lu et al proposed a CNN network to detect the ten different diseases in the rice plant. The deep $\mathrm{CNN}$ with multiple pooling strategies is used for disease detection. The dataset used in this research comprises 500 images of healthy and unhealthy rice plant leaves. The proposed model was 
compared with traditional machine learning methods and achieved the highest accuracy up to $95.48 \%$ [17].

Sladojevic et al proposed a CNN model to detect the diseases in different crops. The proposed model recognized 13 different types of diseases from multiple crops. The dataset was collected from different internet sources. The overall accuracy for the proposed model was $96.3 \%$ [18].

Saleem et al extensively discussed deep learning models used to identify various plant diseases. They have compared a number of commonly known CNN models over the plant disease features [19].

\section{Deep Features and Learning Model}

In the study, a total of 2475 images of pepper leaves with 1478 healthy and 997 bacterial disease, which are among the widely known PlantVillage datasets, have been used. The dataset occupies a total of 23.6MB in memory. Each image data is in color, gray, segmented JPEG format (see Figure 1). The size of the images has been re-adjusted to the $256 \times 256$ px size to match the input of the network. The image given below is a snippet of the raw data-set. Within the scope of the study, the following data were given as input to the network (CNN model) in gray level, color level, and segmented forms. Segmentation [20, 21] may provide better feature characterization by omitting redundant image information from the image. Figure 2 shows healthy and diseased leaf images.
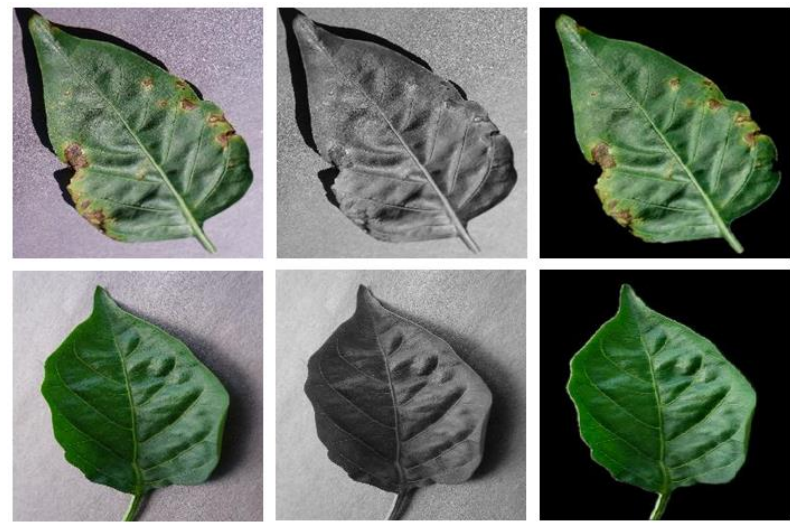

Figure 1. Color, gray and segmented leaf samples (Diseased-top and Healthy-bottom)
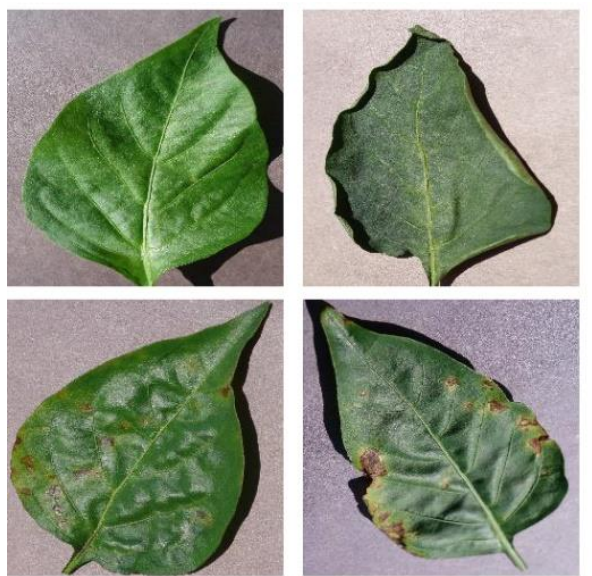
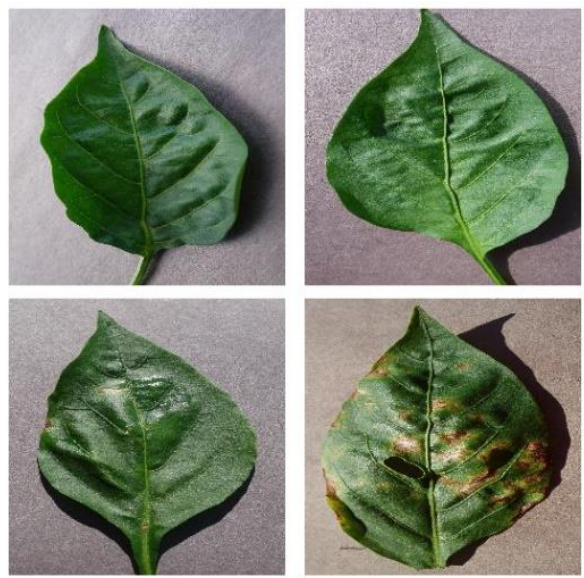

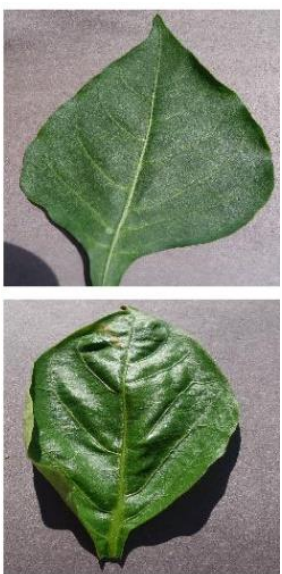

Figure 2. Sample healthy (top) and diseased (bottom) pepper leaves images

Under the concept of deep learning, a deep feature is the consistent response of a unit in the hierarchical model to an input. Convolutional Neural Networks (CNN) have multiple hidden layers and convolution core layers that deepen the network architecture [22, 23]. Features extracted from a CNN network are called "deep features." Deep features are the properties of the data obtained by passing it through a multi-layered artificial neural network and subjecting it to convolution processes. This answer contributes to the decision of the model. Unlike traditional feature extraction methods, deep features are obtained from fully connected layers of convolutional neural networks and can be used directly. On the other hand, deep features have been used in many researches and applications instead of features prepared by hand recently. Studies have proven that powerful features can be extracted using deep learning for operations such as object recognition and tracking. Thanks to these attributes, high success has been achieved in many applications such as object recognition, classification or tracking.
DarkNet-19 model, which is a pre-trained convolutional neural network, has been used in this study. It is a new generation network that has been developed recently. This architecture has a 19-layer network structure and 256x256 sized images are given as input to the network. It is a convolutional neural network model developed on the basis of YOLOv2. Like the VGG model, it usually operates with a $3 \times 3$ filter. It increases the number of channels to two after each pooling step. It uses $1 \times 1$ filters to compress the global average pooling and feature representation to make prediction in the network. The DarkNET exhibits remarkable performance on the real time object detection. The network handles the object detection as a simple regression problem compare to commonly known CNNs.

In the study, the SoftMAX classifier in the last layer of the network has been removed and the naive bayes, the support vector machine, the nearest neighbor and decision tree classifiers have been replaced with it. The features obtained from the fully connected layer have been given directly to these classifiers. Classifiers have been used with their default parameters and no optimization has been made. 
Raw images, gray-level images and color-level segmented images in the data-set were given separately to the pretrained DarkNET-19 network. Performance results have been obtained as a total of 9 test results for all three data and using all three classifiers. The overall proposed model is given in the following Figure 3.

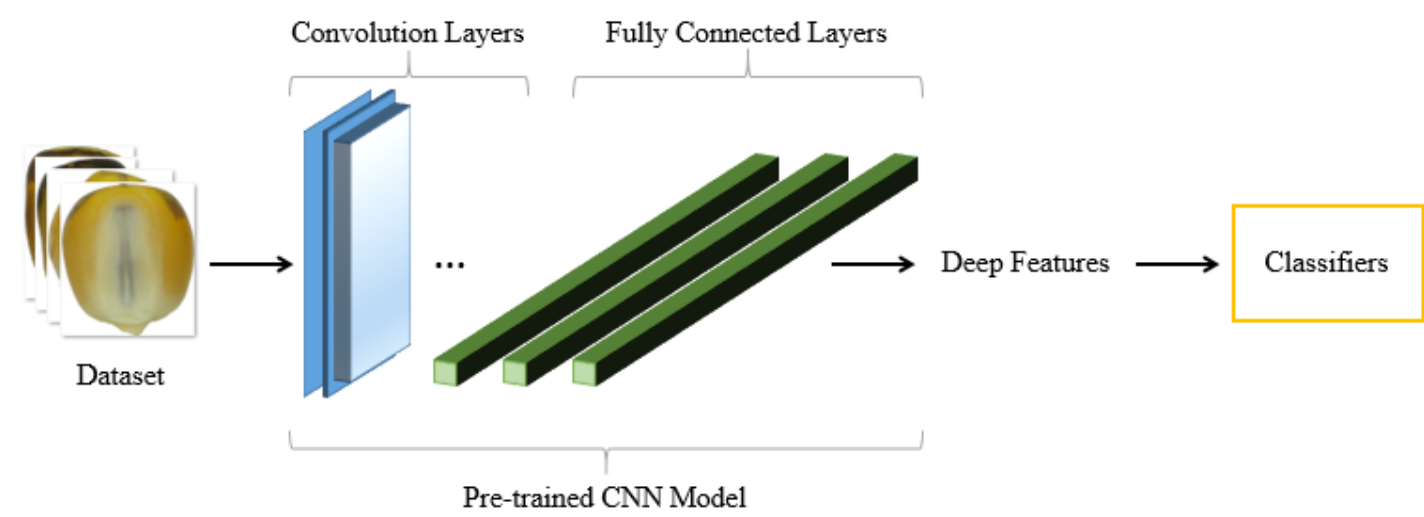

Figure 3. The overall structure of the learning model

The Softmax classifier used in the last layer of the deep learning network is the most basic/primitive classifier known in the literature. The Softmax activation function simply provides normalizing the outputs by converting a number vector to a probability vector. Therefore, four different advanced classifiers have been used instead of SoftMax classifier. These classifiers are Gaussian based Naïve Bayes, Linear Support Vector Machine, K-Nearest Neighbor and Decision Trees. The utilized classifiers are commonly known methods in the literature. Beside the classifiers, Principal Component Analysis (PCA) method has been used to reduce the dimension of the features. The PCA has been utilized after feature extraction process is completed in the last layer (the classification) of the network. By using it classification process has been made faster. Since the required time to process features decreased because of dimension reduction.

\section{Experiments}

The hardware utilized for the experiments has a 6th generation I7 CPU, 8GB RAM, 2GB GTX750 GPU, and 240GB SSD HDD. Experiments have been performed with MATLAB (2020a) environment. The gray-level, colorlevel and segmented images in the data-set have been given separately to the input of pre-trained DarkNET-19 network. Performance results four classifiers were used for each of the three data sets. For each classifier, observation was made when the PCA was active / inactive. Finally, a total of 24 test results were obtained. The Table 1 demonstrates the feature extraction time on the processed data.

Table 1 Feature extraction time

\begin{tabular}{lcc}
\hline CNN Model & $\begin{array}{c}\text { Feature } \\
\text { Number }\end{array}$ & $\begin{array}{c}\text { Feature Acquiring } \\
\text { Time }\end{array}$ \\
\hline DarkNet-19 & 1000 & $382,86 \mathrm{~s}$ \\
\hline
\end{tabular}

Gaussian Naïve Bayes (Gaussian NB), Linear Support Vector Machine (Linear SVM), K-Nearest Neighbor (KNN) and Decision Tree (DT) classifiers have been used in the experiments. These classifiers are applied to all of the features and to a certain part of the features reduced using the Principal Component Analysis (PCA).

Performance metrics used in the study are given in equation (1), (2), (3) and (4). The overall classification performance on the data is expressed with the accuracy (Acc.) metric. The success of finding negative samples in the data is expressed with the precision (Pre.) metric. The success of finding positive samples in the data is expressed with the sensitivity (Sen.) metric. Finally, the harmonic mean of the precision and sensitivity metrics are found with the F-Score metric. This metric is mainly used to avoid making an incorrect model selection in non-uniform data sets.

$$
\begin{gathered}
\text { Accuracy }=\frac{\mathrm{TP}+\mathrm{TN}}{\mathrm{TP}+\mathrm{FP}+\mathrm{FN}+\mathrm{TN}} \\
\text { Precision }=\frac{\mathrm{TN}}{\mathrm{TN}+\mathrm{FP}} \\
\text { Sensitivity }=\frac{\mathrm{TP}}{\mathrm{TP}+\mathrm{FN}} \\
\mathrm{F}-\text { score }=\frac{2 \mathrm{TP}}{2 \mathrm{TP}+\mathrm{FP}+\mathrm{FN}}
\end{gathered}
$$

In the Table 2 below, the complexity matrices and performance metrics obtained by using four different classifiers of the features obtained with the gray level data set are given. In addition, the dimensionality of the data has been reduced by PCA method and re-classified in each classification method. When the obtained results have been examined, the highest accuracy has been obtained with the Linear SVM classifier as $93.5 \%$. The second and third highest accuracies have been observed with Linear SVM + PCA and KNN classifiers as $93.1 \%$ and $90.5 \%$, respectively. As a result of dimensionality reduction with PCA, the Linear SVM classifier still performed remarkably. On the other hand, it has been determined that the use of PCA significantly has reduced performance in other classifiers. The highest scores in terms of accuracy, precision and sensitivity have been also obtained with the linear SVM classifier. 
Table 1 Classification results of gray-level data based on DarkNET-19 features

\begin{tabular}{lccccccccc}
\hline CNN Model & TP & FP & FN & TN & Acc. & Pre. & Sen. & F-Score & Time \\
\hline Gaussian NB & 812 & 180 & 185 & 1298 & $85,3 \%$ & 0,88 & 0,82 & 0,82 & $11,22 \mathrm{~s}$ \\
Gaussian NB + PCA & 695 & 232 & 302 & 1246 & $78,4 \%$ & 0,80 & 0,75 & 0,72 & $17,34 \mathrm{~s}$ \\
Linear SVM & 899 & 62 & 98 & 1416 & $\mathbf{9 3 , 5 \%}$ & 0,94 & 0,94 & 0,92 & $25,18 \mathrm{~s}$ \\
Linear SVM + PCA & 880 & 55 & 117 & 1423 & $93,1 \%$ & 0,92 & 0,94 & 0,91 & $20,93 \mathrm{~s}$ \\
K-NN. & 786 & 25 & 211 & 1453 & $90,5 \%$ & 0,87 & 0,97 & 0,87 & $42,57 \mathrm{~s}$ \\
K-NN. + PCA & 335 & 0 & 662 & 1478 & $73,3 \%$ & 0,69 & 1,00 & 0,50 & $19,91 \mathrm{~s}$ \\
DT & 755 & 190 & 242 & 1288 & $82,5 \%$ & 0,84 & 0,80 & 0,78 & $19,59 \mathrm{~s}$ \\
DT + PCA & 699 & 164 & 298 & 1314 & $81,3 \%$ & 0,82 & 0,81 & 0,75 & $18,84 \mathrm{~s}$ \\
\hline
\end{tabular}

In the Table 3 below, the complexity matrices and performance metrics obtained by using four different classifiers of the features obtained with the color-level data set are given. The dimensionality of the data has been reduced by PCA method and re-classified in each classification method. The highest accuracy has been obtained with the Linear SVM classifier as $98.8 \%$. The second and third highest accuracies have been observed with Linear SVM + PCA and KNN classifiers as $98.3 \%$ and
$93.7 \%$, respectively. As a result of dimensionality reduction with PCA, the Linear SVM classifier still performed remarkably. On the other hand, it has been determined that the use of PCA significantly has reduced performance in other classifiers. The highest scores in terms of accuracy and precision the linear SVM classifier is outperformed other classifiers and the highest sensitivity metric value has observed with KNN classifier.

Table 3 Classification results of color-level data based on DarkNET-19 features

\begin{tabular}{lccccccccc}
\hline CNN Model & TP & FP & FN & TN & Acc. & Pre. & Sen. & F-Score & Time \\
\hline Gaussian NB & 877 & 123 & 120 & 1355 & $90,2 \%$ & 0,92 & 0,88 & 0,88 & $9,92 \mathrm{~s}$ \\
Gaussian NB + PCA & 782 & 180 & 215 & 1298 & $84,0 \%$ & 0,86 & 0,81 & 0,80 & $17,38 \mathrm{~s}$ \\
Linear SVM & 976 & 8 & 21 & 1470 & $\mathbf{9 8 , 8 \%}$ & 0,99 & 0,99 & 0,99 & $11,92 \mathrm{~s}$ \\
Linear SVM + PCA & 962 & 8 & 35 & 1470 & $98,3 \%$ & 0,98 & 0,99 & 0,98 & $18,35 \mathrm{~s}$ \\
K-NN. & 844 & 2 & 153 & 1476 & $93,7 \%$ & 0,91 & 1,00 & 0,92 & $39,46 \mathrm{~s}$ \\
K-NN. + PCA & 338 & 0 & 659 & 1478 & $73,4 \%$ & 0,69 & 1,00 & 0,51 & $19,64 \mathrm{~s}$ \\
DT & 869 & 99 & 128 & 1379 & $90,8 \%$ & 0,92 & 0,90 & 0,88 & $17,99 \mathrm{~s}$ \\
DT + PCA & 788 & 141 & 209 & 1337 & $85,9 \%$ & 0,86 & 0,85 & 0,82 & $18,38 \mathrm{~s}$ \\
\hline
\end{tabular}

In the Table 4 below, the complexity matrices and performance metrics obtained by using four different classifiers of the features obtained with the color-level data set are given. The dimensionality of the data has been reduced by PCA method and re-classified in each classification method. The highest accuracy has been obtained with the Linear SVM classifier as $97.7 \%$. The second and third highest accuracies have been observed with Linear SVM + PCA and KNN classifiers as $97.5 \%$ and
$91.1 \%$, respectively. As a result of dimensionality reduction with PCA, the Linear SVM classifier still performed remarkably. On the other hand, it has been determined that the use of PCA significantly has reduced performance in other classifiers. The highest scores in terms of accuracy and precision the linear SVM classifier is outperformed other classifiers and the highest sensitivity metric value has observed with KNN classifier.

Table 4 Classification results of segmented data based on DarkNET-19 features

\begin{tabular}{lccccccccc}
\hline CNN Model & TP & FP & FN & TN & Acc. & Pre. & Sen. & F-Score & Time \\
\hline Gaussian NB & 834 & 157 & 163 & 1321 & $87,1 \%$ & 0,89 & 0,84 & 0,84 & $10,97 \mathrm{~s}$ \\
Gaussian NB + PCA & 731 & 199 & 266 & 1279 & $81,2 \%$ & 0,83 & 0,79 & 0,76 & $19,99 \mathrm{~s}$ \\
Linear SVM & 957 & 16 & 40 & 1462 & $\mathbf{9 7 , 7 \%}$ & 0,97 & 0,98 & 0,97 & $16,56 \mathrm{~s}$ \\
Linear SVM + PCA & 946 & 10 & 51 & 1468 & $97,5 \%$ & 0,97 & 0,99 & 0,97 & $19,43 \mathrm{~s}$ \\
K-NN. & 779 & 3 & 218 & 1475 & $91,1 \%$ & 0,87 & 1,00 & 0,88 & $40,93 \mathrm{~s}$ \\
K-NN. + PCA & 304 & 1 & 693 & 1477 & $72,0 \%$ & 0,68 & 1,00 & 0,47 & $19,43 \mathrm{~s}$ \\
DT & 837 & 119 & 160 & 1359 & $88,7 \%$ & 0,89 & 0,88 & 0,86 & $20,01 \mathrm{~s}$ \\
DT + PCA & 770 & 130 & 227 & 1348 & $85,6 \%$ & 0,86 & 0,86 & 0,81 & $19,46 \mathrm{~s}$ \\
\hline
\end{tabular}


The accuracy performances of the classifiers have been graphically demonstrated for the three (gray, color and segmented) dataset in Figure 4. Ultimately, the best performance has been acquired about $98,8 \%$ on color dataset. The second and third best accuracy performances have been observed on color level image dataset with $98,3 \%$ and segmented image dataset with $93,7 \%$, respectively.

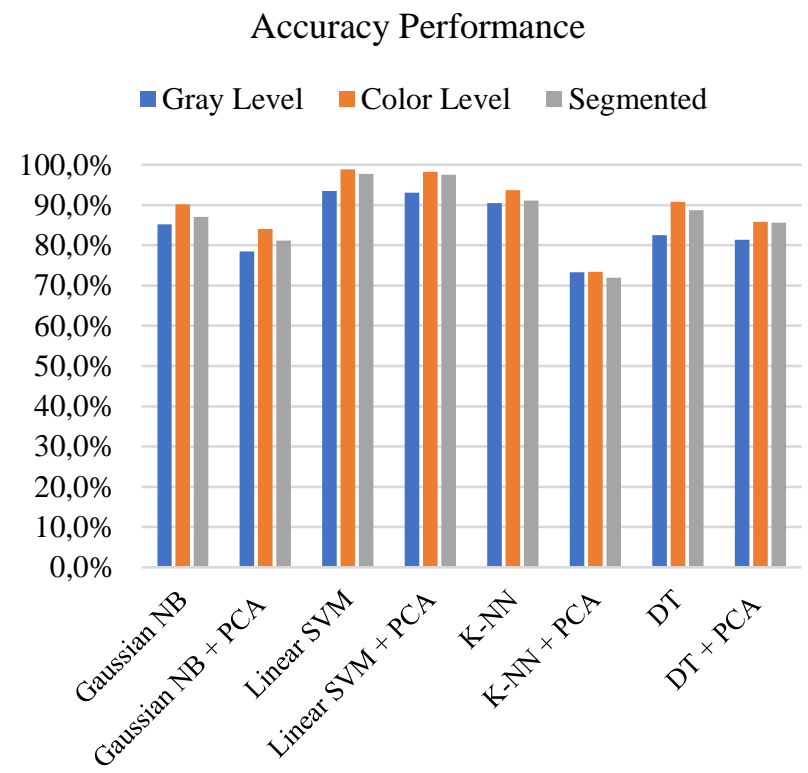

Figure 4. Comparing classifier performances

\section{Conclusion}

In this study, DarkNET-19 pre-trained CNN model has been utilized as feature extractor. The utilized data consists of 2475 images of pepper leaves (1478 healthy and 997 bacterial disease). The dataset consists of gray-level, colorlevel and segmented images of the same leaf data. The features have been acquired from the last fully connected layer of the CNN model. There are four different classifiers utilized as final layer instead of the softmax classifier. The features have been classified with enabling PCA and disabling PCA. It has been determined that the best classifying accuracy was realized with Linear SVM classifier as $98,8 \%$ on the features acquired from the colorlevel images. The highest performance has been observed with linear SVM for all three case (gray, color and segmented). The remaining classifiers have also demonstrated promising performance. It should be noted that there are no optimization or performance enhancing like feature selection, transfer learning or feature fusion. The computer-assisted systems are very useful to identify the class of an object. We have proposed a simple and effective approach to identify the bacterial disease on the pepper leaves. In future, we plan to apply transfer learning and future fusion to enhance and accelerate the existing methods.

\section{Acknowledgements}

We thank Akdeniz and Bandırma 17 Eylül Universities for the basic infrastructure that allowed the development of the study.

\section{References}

[1] S.S. Abu-Naser, K.A. Kashkash and M. Fayyad, "Developing an Expert System for Plant Disease Diagnosis," Journal of Artificial Intelligence, vol. 1, no. 2, pp. 78-85, 2008.

[2] K. Gowthami, M. Pratyusha and B. Somasekhar, "Detection of diseases in different plants using digital image processing," International Journal of Scientific Research in Eng., vol. 2, no. 2, pp. 18-23, 2017.

[3] M.A. Ebrahimi, M.H. Khoshtaghaza, S. Minaei and B. Jamshidi, "Vision-based pest detection based on SVM classification method," Computers and Electronics in Agriculture, 137, pp. 52-58, 2017.

[4] N. Guettari, A.S. Capelle-Laizé and P. CarréBlind, "Image steg analysis based on evidential k-nearest neighbors," IEEE International Conference on Image Processing (ICIP), pp. 2742-2746, 2016.

[5] F. Jobin, D. Anto and K. Anoop, "Identification of leaf diseases in pepper plants using soft computing techniques," pp. 168-173, 2016.

[6] S. H. Lee, C. S. Chan, S. J. Mayo and P. Remagnino, "How deep learning extracts and learns leaf features for plant classification," Pattern Recognition, 71, pp. 1-13, 2017.

[7] K. P. Ferentinos, "Deep learning models for plant disease detection and diagnosis," Computers and Electronics in Agriculture, 145, pp. 311-318, 2018.

[8] M. Islam, A. Dinh, K. Wahid and P. Bhowmik, "Detection of potato diseases using image segmentation and multiclass support vector machine," IEEE 30th Canadian Conference on Electrical and Computer Engineering (CCECE), pp. 1-4, 2017.

[9] Y.C. Zhang, H.P. Mao, B. Hu and M.X. Li, "Features selection of cotton disease leaves image based on fuzzy feature selection techniques," IEEE proceedings of the 2007 international conference on wavelet analysis and pattern recognition, 2007.

[10] S.R. Dubey and A.S. Jalal, "Detection and classification of apple fruit diseases using complete local binary patterns," IEEE 2012 third international conference on computer and communication technology, 2012.

[11] G. Owomugisha and E. Mwebaze, "Machine learning for plant disease incidence and severity measurements from leaf images," 15th IEEE international conference on machine learning and applications, 2015.

[12] A. Meunkaewjinda, P. Kumsawat, K. Attakitmongcol, and A. Srikaew, "Grape leaf disease detection from color imagery using hybrid intelligent system," IEEE 5th Int. Conf. on Electrical Engineering/Electronics. Computer, Telecom. and Inf. Technology, 2008.

[13] M. R. Naik and C.M.R. Sivappagari, "Plant leaf and disease detection by using HSV features and SVM classifier," International journal of engineering science and computing, vol. 6, no. 12, pp. 3794-3797, 2016.

[14] B. Liu, Y. Zhang, D. He and Y. Li, "Identification of Apple Leaf Diseases Based on Deep Convolutional 
Neural Networks," Symmetry, vol. 10, no. 1, pp. 11, 2017.

[15]C. DeChant, T. Wiesner-Hanks, S. Chen, E. L. Stewart, J. Yosinski, M. A. Gore and R.J. Nelson, H. Lipson, "Automated Identification of Northern LeafBlight-Infected Maize Plantsfrom Field Imagery Using Deep Learning," Phytopathology, vol. 107, no. 11, pp. 1426-1432, 2017.

[16] J. Ma, K. Du, F. Zheng, L. Zhang, Z. Gong and Z. Sun, "A recognition method for cucumber diseases using leaf symptom images based on deep convolutional neural network," Computers and Electronics in Agriculture, vol. 154, no. 09, pp. 18-24, 2018.

[17] Y. Lu, S. Yi, N. Zeng, Y. Liu and Y. Zhang, "Identification of rice diseases using deep convolutional neural networks," Neurocomputing, 267, pp. 378-384, 2017.

[18] S. Sladojevic, M. Arsenovic, A. Anderla, D. Culibrk and D. Stefanovic, "Deep Neural Networks Based Recognition of Plant Diseases by Leaf Image
Classification," Computational Intelligence and Neuroscience, 2016.

[19] M.H. Saleem, J. Potgieter and K. M. Arif "Plant Disease Detection and Classification by Deep Learning,". Plants, vol. 8, no. 11, pp. 468, 2019.

[20] E. Dönmez and P. V. Zadeh "A modified graph based approach for leaf segmentation with GPGPU support," 23nd Signal Processing and Communications Applications Conference (SIU), pp. 1797-1800, 2015.

[21]E. Dönmez and A. F. Kocamaz, "A Hog \& Graph Based Human Segmentation from Video Sequences," International Conference on Artificial Intelligence and Data Processing (IDAP), pp. 1-5, 2018.

[22]E. Dönmez, "Discrimination of Haploid and Diploid Maize Seeds Based on Deep Features," 28th Signal Processing and Communications Applications Conference (SIU), pp. 1-4, 2020.

[23]E. Dönmez, "Classification of Haploid and Diploid Maize Seeds based on Pre-Trained Convolutional Neural Networks," Celal Bayar University Journal of Science, vol. 16, no. 3, pp. 323-331, 2020. 